\title{
A Plasma Reactor for Experimental Investigation of Sterilization Processes: Preliminary Results on Escherichia Coli
}

\author{
Alessandra Decina ${ }^{1}$, Annunziata D’Orazio $^{2 *}$, Riccardo Barni ${ }^{1}$, Alessandra Polissi $^{3}$, Claudia Riccardi $^{1}$ \\ ${ }^{1}$ Dipartimento di Fisica G. Occhialini, Università degli Studi di Milano-Bicocca, p.za della Scienza 3, Milano I-20126, Italy \\ ${ }^{2}$ Dipartimento di Ingegneria Astronautica, Elettrica ed Energetica, Sapienza University of Rome, Via Eudossiana 18, Roma \\ 00184, Italy \\ ${ }^{3}$ Dipartimento di Biotecnologie e Bioscienze, Università degli Studi di Milano-Bicocca, p.za della Scienza 3, Milano I-20126, \\ Italy
}

Corresponding Author Email: annunziata.dorazio@uniroma1.it

https://doi.org/10.18280/ijdne.160305

Received: 17 August 2020

Accepted: 25 April 2021

\section{Keywords:}

plasma sterilization process, hydrogen peroxide plasma, argon plasma, Escherichia coli, precursor gas, emission spectra, survival curves, risk assessment

\begin{abstract}
Plasma process is a promising physical method for sterilization. Due to the lack of suitable plasma diagnostics, installed in the reactors, a general lack of knowledge happens about the gas-phase chemical composition and the influence of operating parameters. The same lack of knowledge happens in case of gas plasma generated from different gases, candidates for sterilization purposes. The aim of this work is to understand the role of different agents acting in the sterilization process, evaluate the effectiveness of different precursor gases and validate the plasma reactor dedicated. A plasma reactor, equipped with an extended set of plasma diagnostics is dedicated to the understanding the role of different agents of gas plasma sterilization process. A microbiological investigation is carried out to evaluate the efficiency of the steps of the process on cultures of Escherichia coli, using both hydrogen peroxide and argon. The effects on the colonies of Escherichia Coli are assessed in terms of species present in the chamber, acquiring the spectra resulting from the exposure of the Petri dish to sterilization. With regard to the sterilization action of Peroxide and Argon plasma, CFUs are counted for each significant point of the cycle and survival curves are provided. Differences emerge between Peroxide and Argon plasma, for action mechanisms and sterilization time. The survival curves show a more effective sterilizing action of Argon plasma. A validation is necessary by an appropriate statistic and by considering more resistant microorganisms. Reactor will be used to evaluate different precursor gases and microorganisms. The use of noncorrosive and non-irritating gas, such as argon, for sterilization purpose implies a simplification of the plant equipment. It would be possible to remove the components for the vaporization and concentration of peroxide, and to eliminate the management of the boxes containing discarded peroxide, managed to date as hazardous wastes and to reduce significantly the risk for health professionals.
\end{abstract}

\section{INTRODUCTION}

In modern medicine and in the pharmaceutical industry, as well as in other sectors, the efficient sterilization of tools and surfaces is of central importance [1]. The main challenge for these applications is selecting a reliable sterilization agent. Currently, sterilization methods based on gas-phase are drawing significant interest, since a large number of critical devices (according to the classification of Spaulding 1972 [2]) cannot undergo thermal treatments. In this framework the use of hydrogen peroxide as chemical decontaminant and sterilant is widely spread, mainly because its simplicity and its environmentally friendly decontamination products, water vapor and oxygen [3]. In the past decade, decontamination by hydrogen peroxide has been used frequently for sterilization purposes in a wide range of applications [4]. Plasma process is considered a promising physical method for sterilization [5]. It is generally performed producing oxidizing radicals in the plasma state, starting with oxygen or water vapor in the gas phase mixture feeding the electrical discharge [5]. Within this approach, the prospect of plasma sterilization employing directly hydrogen peroxide was proposed $[6,7]$ and applied as a commercial sterilization device [8].

Despite the hydrogen peroxide plasma sterilization process and its applications were well characterized in literature, little is known concerning such plasmas [9, 10]. Most of the modern research focused on the production of hydrogen peroxide, mainly from water vapor and using atmospheric pressure plasmas [11-15]. No data exist so far in literature, at the best of our knowledge, concerning key physical plasma parameters, even like the electron temperature and density, not to mention the flux and energy of ions impinging on an exposed surface. No one could find information about the gas-phase chemical composition, like the dissociation degree or the density and fluxes of (oxidizing) radicals towards such surfaces or the UV emission. Even more disturbing it lacks data showing how the plasma state is influenced by operating parameters like discharge power level, pressure range and so on. This is mainly due to the lack of suitable plasma diagnostics installed in the reactors used in the processing. In general, the poor 
knowledge, control and understanding of plasma processes, explain why, despite their promising properties and the claims so abundant in scientific literature, rarely they find real applications. The same lack of knowledge happens in case of the gas plasma generated from other gases that could be good candidates for sterilization purposes. Recent papers discuss about the existence of a 'true' plasma decontamination processes [16]. An evaluation is performed about the role of wavelength radiation for the sporicidal effectiveness [17]. A comparison is made between Oxygen and Argon in terms of power and exposure time with regard to Escherichia coli [18]. A comparison is made between Oxygen, Nitrogen and Argon with regard to $\mathrm{P}$. aeruginosa or $\mathrm{S}$. aureus and $\mathrm{B}$. subtilis spores [19].

In this work we present preliminary results on the plasma sterilization obtained by means of dedicated plasma reactor, equipped with an extended set of plasma diagnostics, including RF electrical probes, movable Langmuir probes, optical emission spectroscopy (OES) with a spectrometer and photomultiplier tube (PMT), as described in a previous paper [16]. We carried out a microbiological investigation, to evaluate the efficiency of the various steps of the process on cultures of Escherichia coli, using both hydrogen peroxide and argon, an inert gas, until it is not dissociated by the action of radiofrequency discharge. We present the results of the physical characterization of the process, carried out by measurement of pressure and temperature, and by identification of detected chemical species, and their interpretation by considering of the survival curves of microorganisms. A comparison is made between argon and hydrogen peroxide that currently is the only gas-plasma used in hospitals for sterilization purposes. The use of noncorrosive and non-irritating gas, such as argon, for sterilization purpose implies a simplification of the plant equipment. It would be possible to remove the components for the vaporization and concentration of peroxide. In addition, it would be possible to eliminate the management of the boxes containing discarded peroxide, managed to date as hazardous wastes and to reduce significantly the risk for health professionals. The paper is organized as follows. In section 2 we describe the details of experimental set-up and the steps of the work. More specifically, in section 2.1 we describe the experimental plasma reactor and plasma diagnostic equipment, in section 2.2 the process of treatment of selected microorganism; in section 2.3 we describe the preliminary tests; in section 2.4 the chemical species for the different precursor gases are identified when the microorganisms are not considered; in section 2.5 the experimental trials performed with microorganism populations in case of different precursor gases are described. In section 3 we present and discuss experimental results, in terms of chemical species detected into the chamber, due to the interaction of plasma and biological matter (section 3.1), and in terms of survival curves (section 3.2). In section 4 we discuss the efficacy of precursor gases by comparing results. section 5 is devoted to conclusions.

\section{MATERIALS AND METHODS}

\subsection{Experimental set-up}

The experimental set-up, for which a lay-out is displayed in Figure 1, consists of a cross-shaped vacuum chamber equipped with a RF plasma source [16].

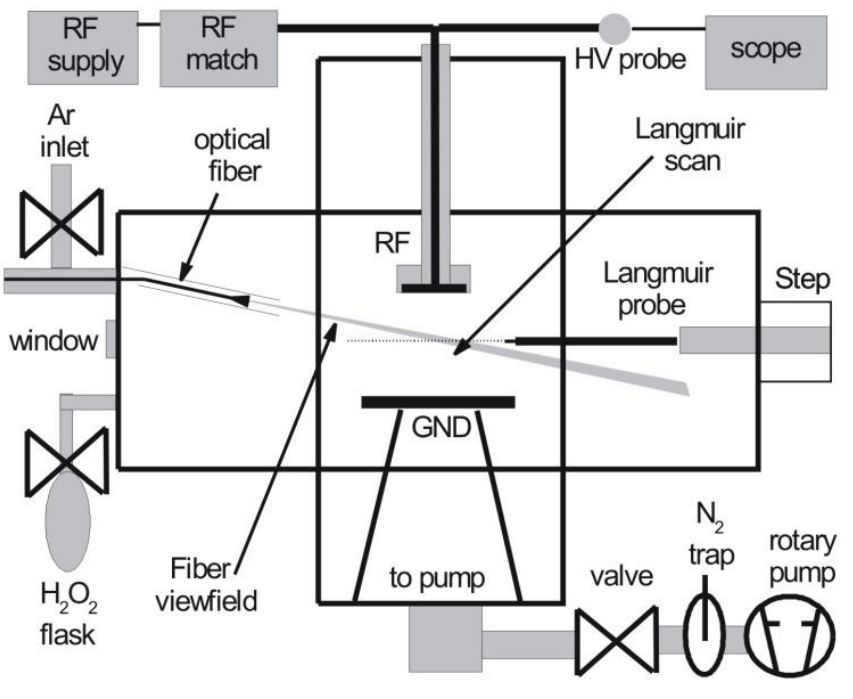

Figure 1. Schematic view of the experimental setup and diagnostics of the plasma reactor. The optical fiber has been connected to a UV-Vis spectrometer or to a phototube.

The chamber, with diameter $100 \mathrm{~mm}$ and two arms size 215 $\mathrm{mm}$ long, has several ports on the four CF-100 flanges and can be evacuated by a rotary pump, up to a residual pressure of $3.3 \times 10^{-1} \mathrm{~Pa}$. The chamber volume was estimated to be $3.4 \mathrm{~L}$. A constant flow of Ar or He could be provided and controlled by a flow meter, whereas hydrogen peroxide in a sealed Pyrex bottle is evaporated into the chamber through a micrometer needle valve. Total pressure in the chamber is measured by a Pirani gauge. The chamber could be considered as a wellmixed reactor [20-23]. The RF source consists of a stainlesssteel disk, diameter $30 \mathrm{~mm}$ and thickness $2 \mathrm{~mm}$, coupled through a vacuum feed-through on the top flange to a semiautomated RF matching unit, which could be fed by a 13.56 $\mathrm{MHz}$ RF supply. The mainly capacitive RF coupling of the discharge is enhanced by the presence of a counter-electrode, a stainless-steel disk, diameter $80 \mathrm{~mm}$ and thickness $2 \mathrm{~mm}$, facing the source at a fixed distance, typically $43 \mathrm{~mm}$, and working as a support for the samples to be processed.

Whereas on the front flange a $100 \mathrm{~mm}$ quartz window is mounted, on the rear flange three vacuum ports accommodates the two gas-inlets and an optical fiber vacuum feed-through. Hydrogen peroxide was prepared from a commercial waterperoxide solution (130 vol. corresponding to $35 \mathrm{wt}$ \%). Thanks to the lower vapor pressure of hydrogen peroxide (180 $\mathrm{Pa}$ vs $2470 \mathrm{~Pa}$ at $20^{\circ} \mathrm{C}$ [24]), the solution concentration could be raised by distillation, pumping away the mainly water vapor gas-phase in a sealed bottle. About $30 \mathrm{~g}$ of the solution $(11 \mathrm{~g}$ of $\mathrm{H}_{2} \mathrm{O}_{2}$ ), were reduced to $4.64 \mathrm{~g}$, which were used during the experimental campaign.

A concentration of $85 \pm 5$ wt. \% was estimated by repeatedly measuring the equilibrium vapor pressure at $20^{\circ} \mathrm{C}$ [24]. For each experiment, a fixed quantity (about $40 \mu \mathrm{L}$ ) of the enriched solution was put by a syringe in a sealed bottle, and frozen by immersion in a liquid nitrogen Dewar. Then residual air in the bottle was pumped away before melting occurs. Subsequently the whole volume of the peroxide drop is evaporated into the main chamber. After a brief pumping, the chamber is isolated. This allows to reach a gas phase in the reactor at a fixed pressure (about $50 \mathrm{~Pa}$ in these experiments) with a fairly high concentration.

As a diagnostic tool of the plasma state, we employed Optical Emission Spectroscopy (OES). OES of the discharge 
could be used to gain insight in the excited species produced in the source $[25,26]$. Light is collected inside the vacuum chamber with a $50 \mu \mathrm{m}$ UV enhanced fiber optics the imaged plasma volume is approximately constant under the different discharge conditions and the changes in light intensity can be traced to those in the plasma state [20]. Light is delivered through a vacuum feed-through and another fiber to a wide band, low-resolution spectrometer, with a holographic grating 600 lines $/ \mathrm{mm}$ blazed at $400 \mathrm{~nm}$ ) which allows the measurements of the emission spectra. Spectra cover the UVVis-NIR range from 175 to $1100 \mathrm{~nm}$ with a resolution of about $0.6 \mathrm{~nm}$. Line intensity was measured and emitting atoms and molecules have been identified [27, 28].

\subsection{Pathogenic microorganisms}

Bacteria and spores are the pathogens most responsible for nosocomial infections related to surgical instrumentation [1, 16]. The spores are microorganism most resistant to sterilization, also with regard to plasma sterilization. The mechanisms of interaction between plasma and microorganisms depend on many factors, such as the mode of plasma generation, the type of precursor gases, the mode of exposure of the biological sample (direct, in the plasma generation area, or in the afterglow, that is in a remote area in which the sample is invested by the generated particles).

The models developed over the years, regarding the possible mechanisms of interaction between plasma and living matter, in particular the cell membrane, describe the role of the different species generated by dissociation of the precursor gas. More specifically, they take in account the role of free radicals, charged particles (electrons and ions), emitted UV radiation, both of conventional type and in vacuum (VUV), and the synergies with the formation of biochemical complexes that lead to DNA damage [29-38].

In this work, we used cultures of Escherichia coli, a Gramnegative rod-shaped bacterium, both aerobic and anaerobic. The bacterial culture of Escherichia coli used in the plasma sterilization tests was prepared in microbiology laboratory of the Department of Biotechnology and Biosciences University of Milano - Bicocca. The strain used is K-12 MG1655, grown in a suspension with minimal broth $\mathrm{M} 9$, a $\mathrm{NaCl}$ concentration equal to $0.9 \%$ and an addition of Trehalose in concentrations of $250 \mathrm{mmol} / \mathrm{l}$.

Once the Petri dishes were extracted from the reactor, we used the process of the viable count by serial dilutions to estimate the number of CFU surviving to the treatment and plot the survival curves in order to evaluate the effectiveness of the process. In this procedure, the culture on the Petri dish is suspended in $1 \mathrm{~mL}$ of sterile physiological solution; $0.1 \mathrm{ml}$ of this solution, containing the suspended bacteria, are taken and furtherly diluted in $1 \mathrm{~mL}$ of sterile physiological solution, so that the CFU concentration is reduced by an order of magnitude. Again, $0.1 \mathrm{~mL}$ of this solution are diluted in $1 \mathrm{~mL}$ of sterile physiological solution and these steps are replicated up to the detectability limit that in our case is equal to $10^{2} \mathrm{CFU}$ $/ \mathrm{ml}$. All the differently diluted solutions are spread on a Petri dish with agar, which is then placed in incubation at $37^{\circ} \mathrm{C}$. After $24 \mathrm{~h}$ of incubation, the CFUs on the Petri dish are counted, and related to the corresponding dilution level, in order to obtain the value of CFUs number per $\mathrm{ml}$. The working hypothesis of this method is that every vital bacterium gives rise to one and only one $\mathrm{CFU}$.

\subsection{Characterization of the chamber and set of working parameters}

For the choice of test conditions, first we proceeded to determine the heating profiles of the Petri dish during the RF excitation, with measurements of temperature by means of a thermocouple clamped to the Petri dish. The evaluation of the temperature profile as a function of the time aims to identify the radiofrequency power properly, in order to refer the killing of the bacteria to the dissociation of the precursor gas $\mathrm{H}_{2} \mathrm{O}_{2}$ and not to thermal effects. In fact, both the temperature increase that the dissociation degree of the precursor gas is directly proportional to the power used. The temperature rise was recorded for duration of RF excitation equal to 5 minutes and by increasing the RF power in the range $70 \div 300 \mathrm{~W}$ with increments of $25 \mathrm{~W}$. At the end of each cycle of generation and maintaining of the plasma in the RF, the reaction chamber was cooled to an initial temperature equal to $26^{\circ} \mathrm{C}$, before of the subsequent detection with power increase. To facilitate the heat exchange and the cooling process between a test and the other, the pressure of the gas after the switching-off of the RF excitation was brought around to 3 mbar with the introduction of argon.

The temperature values as a function of the time, for different excitation powers, are shown in Figure 2, together with the maximum temperature $\mathrm{T}_{\mathrm{cmax}}$ currently reached in the devices on the market (approximately $50^{\circ} \mathrm{C}$ ), and the maximum temperature $T_{b}$ at which bacteria can survive for a limited time.

Based on the obtained results we decided to carry out the plasma process with an exposure time $t_{\exp }=5 \mathrm{~min}$, and the frequency $\mathrm{f}$ and the power $\mathrm{P}$ of RF excitation equal to 13.56 $\mathrm{MHz}$ and $100 \mathrm{~W}$ respectively. In this way, the maximum temperature reached for the duration of the plasma state is maintained below the value $\mathrm{T}_{\mathrm{cmax}}$.

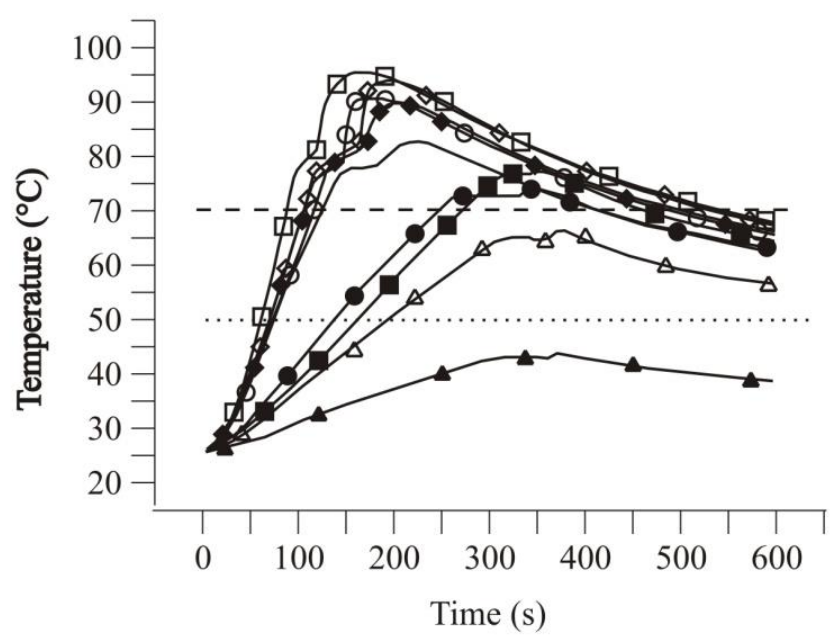

Figure 2. Heating curves of the Petri dish during plasma excitation

\subsection{The chemical species with no bacteria}

The chemical species in the reaction chamber after the dissociation of the precursor gas induced by the RF excitation were identified by optical emission spectroscopy that allows to identify only the optically active species. The tests with no bacteria were made for different powers in the range $70 \div 300$ $\mathrm{W}$, with increments of $25 \mathrm{~W}$, in order to understand the effect 
of excitation power on the dissociation degree of the precursor. The precursor gases under investigation were argon and hydrogen peroxide. A more detailed description of the bulk plasma volume and the electron temperature across the plasma can be found in the ref. [20].

Among the two radical species generated by the dissociation of $\mathrm{H}_{2} \mathrm{O}_{2}, \bullet \mathrm{OH}$ and $\mathrm{HO}_{2} \bullet$, only the first is optically active and therefore detectable by optical emission spectroscopy.

The increase of the power implies an increase of the intensity of the peaks, corresponding to an increase of the concentration of the dissociation products, and a growing number of peaks in the range 280-600 nm, typical of diatomic oxygen $(385.92 \mathrm{~nm}), \mathrm{CO}_{2}(325.85 \mathrm{~nm})$ and $\mathrm{CO}(519 \mathrm{~nm})$.

The presence of the groups of $\mathrm{CO}$ and $\mathrm{CO}_{2}$ increases with the power, and is due to the possible presence in the chamber of organic residues, related to the handling and lubrication of building elements during construction of the plant. The increase of concentration of iron with the RF power is linear, and probably due to a phenomenon of etching caused by the ionized particles of the plasma towards the electrodes. Even the increase of concentration of ROS (Reactive Oxygen Species) and $\mathrm{H}$, species responsible of the etching of cell membranes and therefore fundamental elements to ensure the sterility conditions, is linear with the excitation power. We do not report here the described results, while Figure 3 (a) shows the spectrum relative to the power of $100 \mathrm{~W}$, selected for the experiments.

When using argon as precursor gases, the generated species are very different with respect to the previous case. The optically active species detected by spectroscopy are $\mathrm{OH}$ and $\mathrm{H}$, from water vapor present in the reactor, the ion $\mathrm{Ar}^{+}$arising from the dissociation of argon, the argon with all its emission lines, and $\mathrm{CO}$ (also in this case is presumably due to traces of organic material remaining in the chamber) that appears only for higher powers.

Concentration of hydrogen increases almost linearly with the RF- power, for values up to $175 \mathrm{~W}$, while for the highest values it presents saturation. The trend of $\mathrm{OH}$ is less regular, presenting a minimum for the power ranging between 100 and $150 \mathrm{~W}$, a maximum at about $175 \mathrm{~W}$ and a subsequent minimum at about $250 \mathrm{~W}$. Also for $\mathrm{CO}$ occurs a maximum of the concentration at $175 \mathrm{~W}$, a minimum at about $200 \mathrm{~W}$ and a second maximum in the range $225 \div 300 \mathrm{~W}$.

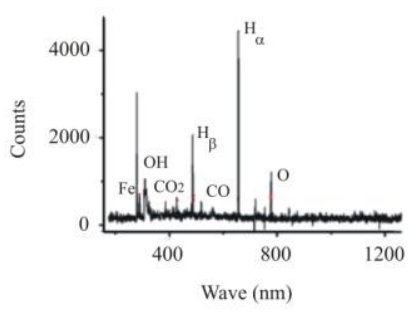

(a)

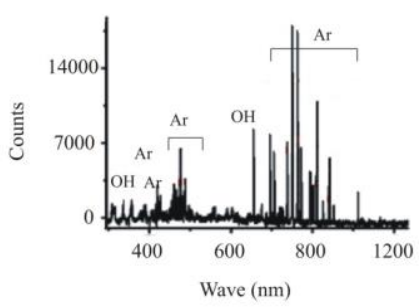

(b)
Figure 3. Chemical species in the reaction chamber in absence of bacteria after dissociation of the peroxide (a) and Argon (b)

As regards the concentration of Argon, for all emission lines, a maximum occurs at $175 \mathrm{~W}$. For each emission line of Argon, the intensity values change with the RF power between 20 and $30 \%$. This means that the argon plasma generates the detected species, over the whole range of powers, in equal amounts, while the fraction of power, not useful to the generation of these species, is dissipated in the plasma sheath near the electrode or in the electrode itself. Also for Argon, we report in Figure 3 (b) the spectrum relative to the power of $100 \mathrm{~W}$, selected for the experiments, and we do not report here all the described results.

The difference between the active species, generated in the plasma state, implies that peroxide hydrogen and argon realize their sterilizing action through different mechanisms.

While the $\mathrm{OH}$ concentration remains the same for the two cases, argon shows a concentration of photons in the range of $\mathrm{UV}$ much greater compared to $\mathrm{H}_{2} \mathrm{O}_{2}$. The $\mathrm{Ar}^{+}$ion, which can make the etching of the cell membranes more efficient, is present only in the case of argon as a precursor. Atomic oxygen, characterized by a high oxidizing power and, therefore, an intense etching activity on biological components, is present only in the case of peroxide as a precursor. Therefore, sterilization with argon is most based on inactivation by UV radiation, and less on the etching of biological components, while the reverse happens for process with $\mathrm{H}_{2} \mathrm{O}_{2}$, which is based on the oxidant power of oxygen.

\subsection{Microbiological study}

For the present work, we prepared some bacterial cultures with strains of Escherichia Coli in saline. Ascertained during the tests that this type of configuration presents problems for bacterial survival, due to drying of the solution (required to avoid being empty, cooling due to evaporation of the water and the consequent damage to the internal cellular structures for freezing water), we realized the bacterial cultures in saline solution with addition of Trehalose ([31] Garcia de Castro et al. 2000). More specifically, it has been realized a suspension of Escherichia coli in saline solution of $0.9 \% \mathrm{NaCl}$ with the addition of $250 \mathrm{mmol}$ of Trehalose.

The initial concentration was set equal to $10^{9} \mathrm{CFU} / \mathrm{ml}$. On each Petri dish has been deposited a quantity of bacterial suspension equal to $100 \mu \mathrm{L}$. All samples have been dried in an oven, at a temperature between 30 and $35^{\circ} \mathrm{C}$. Preliminarily, we assessed the loss of vitality due to the drying and exposure to vacuum. Such tests have indicated a survival to the drying of $10^{7} \mathrm{CFU} / \mathrm{ml}$, while the exposure to vacuum, following the drying, did not cause cell death. These results were verified by means of a control non-dried culture, and a culture dried under hood, at room temperature, with laminar flow and long times.

With regard to the plasma of hydrogen peroxide, we performed the following tests, related to the experimental conditions. At the end of the treatment, the cells were suspended in sterile solution and then cultured on a Petri dish with agar, at $37^{\circ} \mathrm{C}$ for $24 \mathrm{~h}$.

A. Attainment of the vacuum conditions at $5 \times 10^{-4}$ mbar and diffusion of peroxide in the chamber for $5 \mathrm{~min}$, at to $6 \div 7 \mathrm{mbar}$.

B. Attainment of the vacuum conditions at $5 \times 10^{-4} \mathrm{mbar}$, diffusion of peroxide in the chamber for $5 \mathrm{~min}$, at to $6 \div 7 \mathrm{mbar}$, diffusion of peroxide in the chamber for $5 \mathrm{~min}$, at $0.5 \mathrm{mbar}$ (same pressure conditions for the application of radiofrequency excitation).

C. Attainment of the vacuum conditions at $5 \times 10^{-4} \mathrm{mbar}$, diffusion of peroxide in the chamber for $5 \mathrm{~min}$, at to $6 \div 7 \mathrm{mbar}$, discharge of the chamber until $0.5 \mathrm{mbar}$, radiofrequency excitation $(100 \mathrm{~W})$ for $2.5 \mathrm{~min}$.

D. Attainment of the vacuum conditions at $5 \times 10^{-4}$ mbar, diffusion of peroxide in the chamber for $5 \mathrm{~min}$, at to $6 \div 7 \mathrm{mbar}$, discharge of the chamber until $0.5 \mathrm{mbar}$, radiofrequency excitation $(100 \mathrm{~W})$ for $5 \mathrm{~min}$. 
The trials marked A to D have been necessary for evaluating the effectiveness of the sterilization cycle in its meaningful points.

Trial A has allowed to establish the population of microorganisms killed by exposure to the action of the peroxide alone, biocidal also in the gaseous phase not dissociated from the plasma, although with a slower kinetics. Figure 4 (a) reports the realized cycle in terms of pressure in the chamber as a function of the time.

Trial B has allowed to establish the population of microorganisms killed by exposure to the action of the peroxide alone after 10 minutes. The test $\mathrm{B}$ has been necessary to separate the contribution due to the plasma from that of the exposure to the peroxide, which, in case of not complete dissociation due to RF excitation, could maintain its sterilizing effectiveness for Escherichia coli. In this test, in fact, the suspension of Escherichia coli is subjected to the peroxide vapor for a time identical to that at which it will be subjected during the keeping of the peroxide plasma. The peroxide diffusion (lasting 10 minutes) was carried out at the same pressure of introduction of $\mathrm{H}_{2} \mathrm{O}_{2}$ in chamber, about 6 to $7 \mathrm{mbar}$, for 5 minutes, and for the remaining five minutes at the pressure of plasma generation $(0.5 \mathrm{mbar})$. At the time of plasma generation, the pressure is reduced in order to increase the free mean path of the generated species; in this way, phenomena of collision and recombination are limited and this allows to obtain a more stable and uniform plasma. Figure 4 (b) shows the realized cycle.

Trial $\mathrm{C}$ has allowed to establish the population of microorganisms killed by exposure to the plasma of peroxide after the half of the entire time of excitation, in order to obtain an intermediate point for the survival curve of E.C. The corresponding cycle is shown in Figure 4 (c).

Trial D represents the middle of the standard cycle (composed of two consecutive identical cycles): 5 minutes of exposure to the vapors of $\mathrm{H}_{2} \mathrm{O}_{2}, 5$ minutes for generating and keeping the plasma state with the radiofrequency excitation at $100 \mathrm{~W}$. The cycle is reported in Figure 4 (d).

With regard to the plasma of Argon, we performed the following tests, related to the experimental conditions. At the end of the treatment, the cells were suspended in sterile solution and then cultured on a Petri dish with agar, at $37^{\circ} \mathrm{C}$ for $24 \mathrm{~h}$

E Attainment of the vacuum conditions at $5 \times 10^{-4}$ mbar, diffusion of Argon in the chamber for $5 \mathrm{~min}$, at to $6 \div 7 \mathrm{mbar}$, discharge of the chamber until 0.5 mbar, radiofrequency excitation (100W) for $2.5 \mathrm{~min}$.

F Attainment of the vacuum conditions at $5 \times 10^{-4} \mathrm{mbar}$, diffusion of Argon in the chamber for $5 \mathrm{~min}$, at to $6 \div 7 \mathrm{mbar}$, discharge of the chamber until $0.5 \mathrm{mbar}$, radiofrequency excitation $(100 \mathrm{~W})$ for $5 \mathrm{~min}$.

The trials marked $\mathrm{E}$ and $\mathrm{F}$ have been necessary for evaluating the effectiveness of the sterilization cycle in its meaningful points. Figure 5 reports the realized cycle in terms of pressure in the chamber as a function of the time.

Trial E has allowed to establish the population of microorganisms killed by exposure to the plasma of Argon after the half of the entire time of excitation, in order to obtain an intermediate point for the survival curve of E.C. The corresponding cycle is shown in Figure 5 (a).

Trial F represents the middle of the standard cycle (composed of two consecutive identical cycles): 5 minutes of exposure to Argon, 5 minutes for generating and keeping the plasma state with the radiofrequency excitation at $100 \mathrm{~W}$. The cycle is reported in Figure 5 (b).

The trial related to the exposure to gas Argon was not performed, since this inert gas has not interaction with organic matter until it is not dissociated.

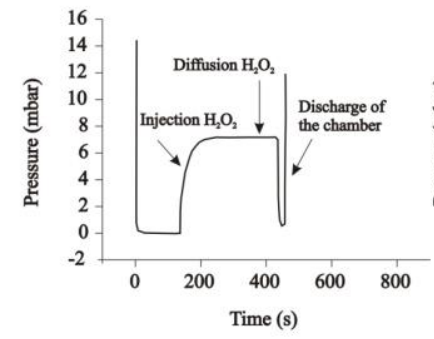

(a)

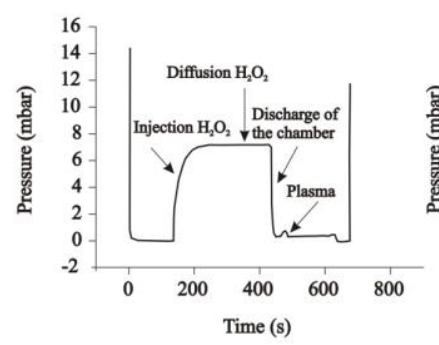

(c)

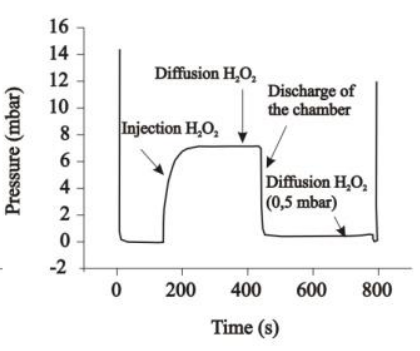

(b)

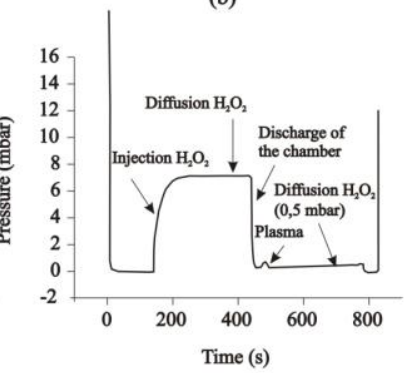

(d)
Figure 4. Sterilization cycles with peroxide: pressure in the chamber as a function of the time for test A (a), B (b) C (c) and $\mathrm{D}(\mathrm{d})$

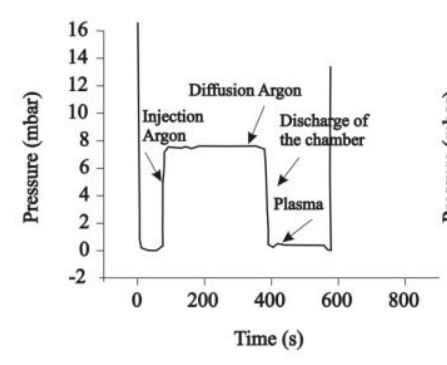

(a)

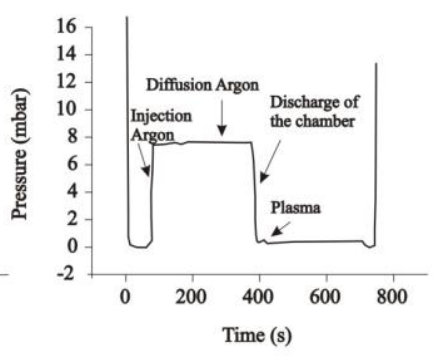

(b)
Figure 5. Sterilization cycles with Argon: pressure in the chamber as a function of the time for test $E$ (a) and $F(b)$

\section{RESULTS}

\subsection{Effectiveness of the process: the detected active species}

To assess the effects of the plasma on the colonies of Escherichia Coli, in terms of species present in the chamber, we acquired the spectra resulting from the exposure of the Petri dish to sterilization. The spectroscopic data were acquired at the beginning of excitation, after 2.5 minutes and at the end ( 5 minutes of exposure). Results are reported in Figure 6.

During the exposure of the bacterial suspension to the plasma of $\mathrm{H}_{2} \mathrm{O}_{2}$, gradually appear species compatible with the etching of cell membranes, such as $\mathrm{CO}$ and $\mathrm{CO}_{2}$, while active species, such as $\mathrm{OH}, \mathrm{H}$ and $\mathrm{O}$, tend to decrease, consumed by the process of interaction with the biological matter. 


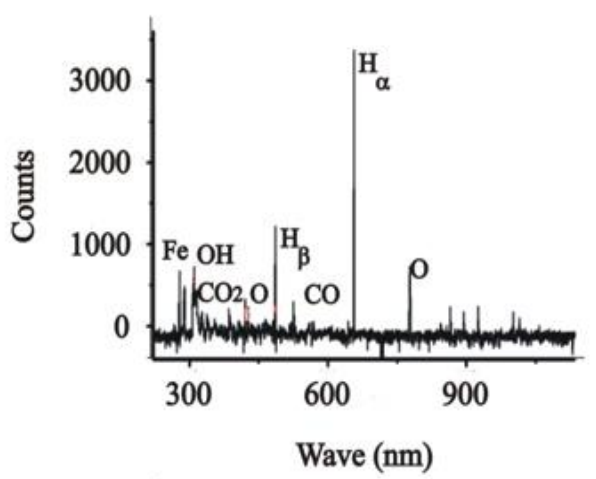

(a)

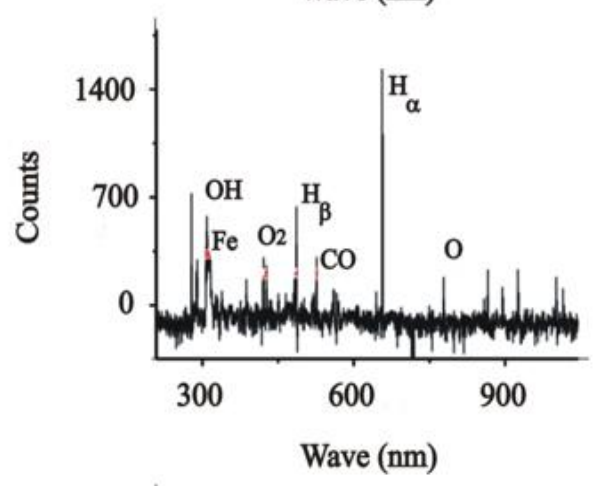

(b)

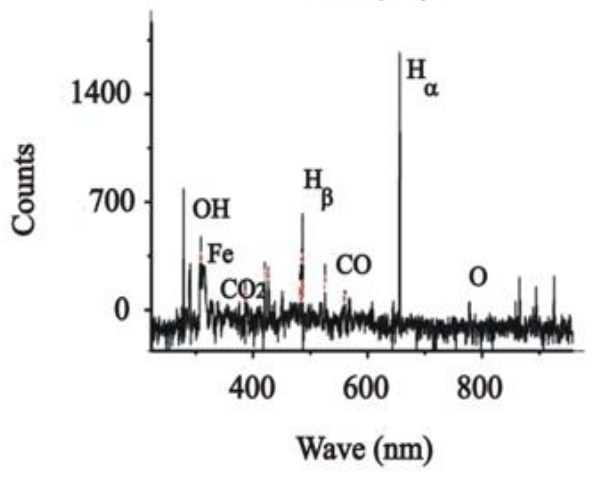

(c)

Figure 6. Chemical species in the reaction chamber with Escherichia Coli after dissociation of the peroxide: beginning of excitation (a), after 2.5 minutes (b) and at the end, after 5 minutes of exposure (c)

Also in case of Argon, to assess the effects of the plasma on the colonies of Escherichia Coli, in terms of species present in the chamber, we acquired the spectra resulting from the exposure of the Petri dish to sterilization. The spectroscopic data were again acquired at the beginning of excitation, after 2.5 minutes and at the end ( 5 minutes of exposure). Results are summarized in Figure 7.

During the exposure of the bacterial suspension to the plasma of Argon, from the first moments of the plasma ignition, the species appear related to organic compounds $\left(\mathrm{N}_{2}\right.$, $\mathrm{N}^{+2}, \mathrm{CO}, \mathrm{CO}_{2}$ ). They however tend all to decrease with the treatment time, as well as tend to decrease the active species consumed by the process of interaction with the biological matter.

\subsection{Effectiveness of the process: the survival curves}

With regard to the sterilization action of the plasma of peroxide, we performed the count of CFUs for each significant point of the cycle, as previously described. Incubation of the suspended cells in a sterile solution after the treatments has provided the following results.

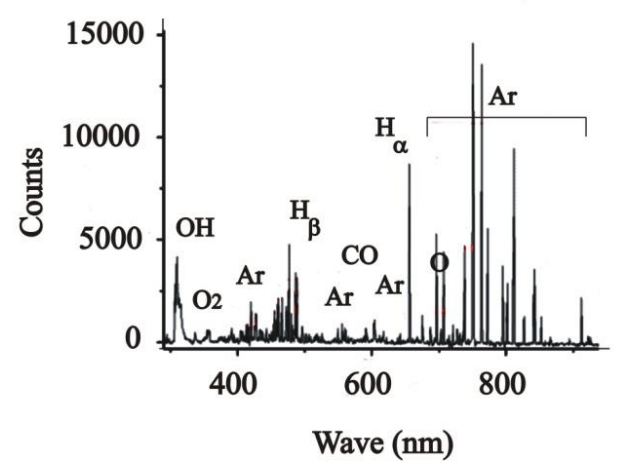

(a)

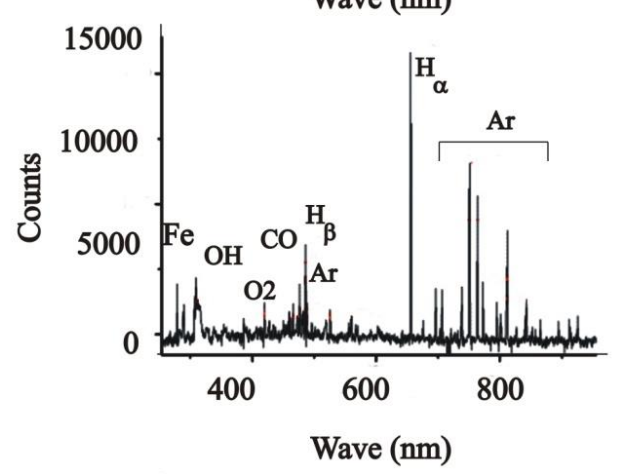

(b)

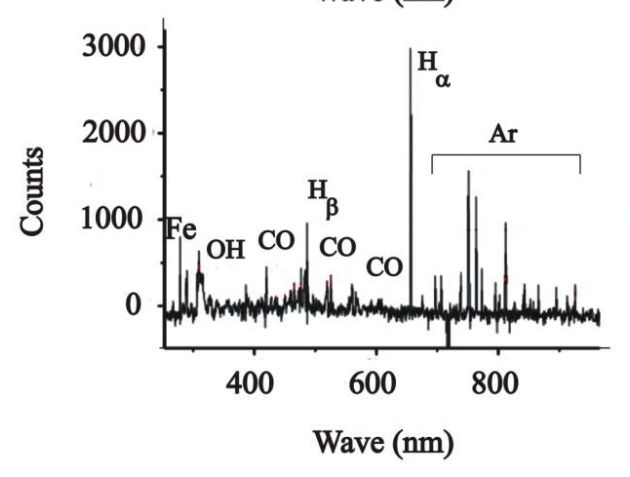

(c)

Figure 7. Chemical species in the reaction chamber with Escherichia Coli after dissociation of argon: beginning of excitation (a), after 2.5 minutes (b) and at the end, after 5 minutes of exposure (c)

Petri A. The exposure for 5 minutes to peroxide in the gas phase of the population of microorganisms has reduced the initial value, equal to $10^{7} \mathrm{CFU} / \mathrm{ml}$ to the final value of $8 \times 10^{6}$ $\mathrm{CFU} / \mathrm{ml}$.

Petri B. The exposure for 10 minutes to peroxide in the gas phase of the population of microorganisms has reduced the initial value, equal to $10^{7} \mathrm{CFU} / \mathrm{ml}$ to the finale value of $1 \times 10^{6}$ $\mathrm{CFU} / \mathrm{ml}$.

Petri C. The exposure for 5 minutes to peroxide in the gas phase of the population of microorganisms and radiofrequency excitation for 2.5 minutes have reduced the initial value, equal to $10^{7} \mathrm{CFU} / \mathrm{ml}$ to the finale value of $1.5 \times 10^{2} \mathrm{CFU} / \mathrm{ml}$. The step of radiofrequency excitation implies a $\log$ reduction of microbial load equal to 5 .

Petri D. After the exposure for 5 minutes to peroxide in the gas phase of the population of microorganisms (starting from the initial value of $10^{7} \mathrm{CFU} / \mathrm{ml}$ ) and to the radiofrequency excitation for 5 minutes it is not detected bacterial growth on the Petri dish (the minimum detectable value for the method of serial dilutions being equal to $10^{2} \mathrm{CFU} / \mathrm{ml}$ ). Therefore, it has been reached the level of SAL required to a sterilization process, i.e. the killing of at least $6 \log$ units of the bacterial load of a microorganism in the half cycle. 
These data allow us to sketch the survival curve of Escherichia coli subjected to the sterilization process with $\mathrm{H}_{2} \mathrm{O}_{2}$ plasma, with a power of RF excitation of $100 \mathrm{~W}$. The curve is shown in Figure $8 \mathrm{a}$ in terms of the number of surviving microorganisms $\mathrm{S}(\mathrm{t})$ as a function of the treatment time. The Sterility Assurance Level (6 log reduction) is obtained with an exposure time of 9 minutes. Neglecting the phase of the peroxide diffusion, which, as shown, doesn't give a decisive contribution to sterilization, one can see from the figure how the process is characterized by two phases. The first phase is characterized by a faster kinetics and is promoted by the action of UV radiation; the coefficient of decimal reduction, i.e. the time required to a reduction of $90 \%(1 \mathrm{log})$ of the initial population, is equal to 30 seconds. The second phase is characterized by a slower kinetics and is promoted by the combined effects of UV radiation, and active species with their action of etching of the cellular membranes; the coefficient of decimal reduction is equal to $1 \mathrm{~min}$ and 36 seconds.

Figure $8 \mathrm{~b}$ shows the number of surviving microorganisms $\mathrm{S}$ (t) during the plasma phase.

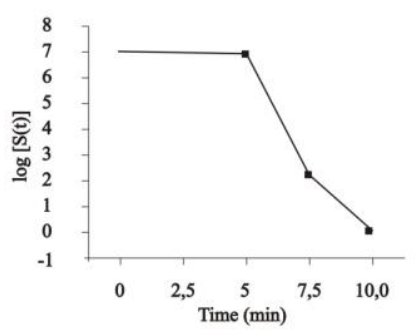

(a)

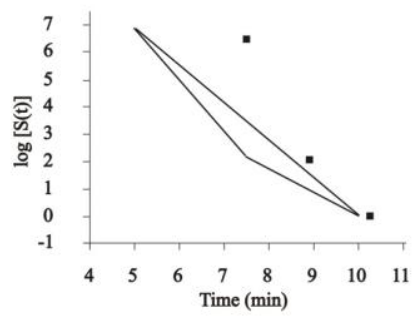

(b)
Figure 8. Survival curve of Escherichia coli subjected to the sterilization process with $\mathrm{H}_{2} \mathrm{O}_{2}$ plasma: diffusion phase and plasma phase in (a), plasma phase in (b). The coefficient of decimal reduction $(1 \mathrm{log})$ of the plasma action is equal to 30 seconds for the first phase and $1 \mathrm{~min}$ and 36 for the second

phase. The SAL (6 log reduction) is obtained in $9 \mathrm{~min}$.

Average mortality rate is obtained as combination of the mortality rates related to the faster and slower kinetic

The number of surviving microorganisms $S(t)$ during the phase of the peroxide diffusion is described by the following differential equation:

$$
\frac{d S}{d t}=-\alpha_{1} S_{0}
$$

where, the coefficient $\alpha_{1}$ represents the cellular mortality due to the action of the peroxide. One can find the number of microorganisms after the time $\mathrm{t}$ for $0 \leq \mathrm{t}<5$ from the following:

$$
S(t)=S_{0} e^{-\alpha_{1} t}
$$

with $\alpha_{1}=0.2$.

From the instant of radio frequency excitations, the diffusion of peroxide has not affect on the microbial reduction, since peroxide is immediately dissociated, to give the plasma state.

The decay of the surviving microorganisms during the phase of plasma, from 5 and 10 minutes, is modeled by means of an average mortality rate $\alpha_{\mathrm{av}}$, obtained as combination of the mortality rates $\alpha_{2}+\alpha_{3}$ and $\alpha_{2}+\alpha_{3^{*}}$, related to the action of UV radiation and charged particles, for faster and slower kinetic respectively. The coefficient $\alpha_{2}$ is constant, since the intensity of UV radiation does not vary in the considered time range, whereas the coefficient $\alpha_{3}$ and $\alpha_{3^{*}}$ are different, since the concentration of the charged particles varies.

The number of surviving microorganisms $\mathrm{S}(\mathrm{t})$ during the phase of the plasma state, for $5 \leq \mathrm{t}<10$, is described by the following equation:

$$
\frac{d S}{d t}=-\alpha_{a v} S_{5}
$$

After the time $\mathrm{t}$ for $5 \leq \mathrm{t}<10$, one can find:

$$
S(t)=S_{5} e^{-\alpha_{a v}(t-\tau)}
$$

with $\alpha_{\mathrm{av}}=1.2$.

Also with regard to the sterilization action of the plasma of Argon, we performed the count of CFUs for each significant point of the cycle, as previously described.

Petri E. After the diffusion of Argon for 5 minutes and the exposure of the population of microorganisms (starting from the initial value of $10^{7} \mathrm{CFU} / \mathrm{ml}$ ) to the radiofrequency excitation for 2.5 minutes, it is not detected bacterial growth on the Petri dish (the minimum detectable value for the method of serial dilutions being equal to $10^{2} \mathrm{CFU} / \mathrm{ml}$ ). Therefore, the level of SAL required to a sterilization process, i.e. the killing of at least $6 \log$ units of the bacterial load of a microorganism in the half cycle, has been reached.

Petri F. After the diffusion of Argon for 5 minutes and the exposure of the population of microorganisms (starting from the initial value of $10^{7} \mathrm{CFU} / \mathrm{ml}$ ) to the radiofrequency excitation for 5 minutes, it is not detected bacterial growth on the Petri dish. The treatment produced identical results with respect to previous case.

These data allow us to sketch the survival curve of Escherichia coli subjected to the sterilization process with Argon plasma, with a power of RF excitation of $100 \mathrm{~W}$. The curve is shown in Figure $9 \mathrm{a}$ in terms of the number of surviving microorganisms $\mathrm{S}(\mathrm{t})$ as a function of the treatment time.

Also for the case of sterilization with plasma of Argon, one can see from the figure how the process is characterized by two phases.

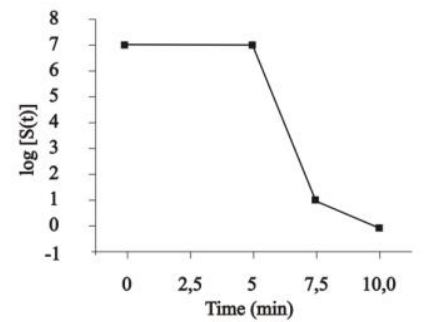

(a)

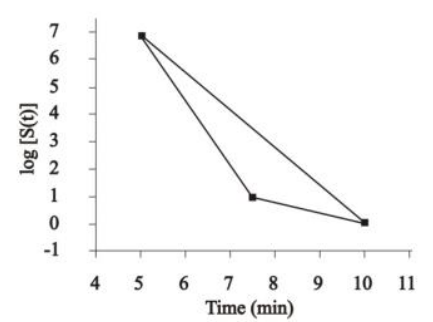

(b)
Figure 9. Survival curve of Escherichia coli subjected to the sterilization process with Argon plasma: diffusion phase and plasma phase in (a), plasma phase in (b). The coefficient of decimal reduction $(1 \mathrm{log}$ ) of the plasma action is equal to 24 seconds for the first phase and 2 min and 30 for the second phase. The SAL (6 log reduction) is obtained in $7.5 \mathrm{~min}$. Average mortality rate is obtained as combination of the mortality rates related to the faster and slower kinetic 
The first phase is characterized by a faster kinetics and is promoted by the action of UV radiation; the coefficient of decimal reduction, i.e. the time required to a reduction of $90 \%$ $(1 \mathrm{log})$ of the initial population, is equal to 24 seconds.

The second phase is characterized by a slower kinetics and is promoted by the combined effects of UV radiation, and active species with their action of etching of the cellular membranes; the coefficient of decimal reduction is equal to 2 min and 30 seconds. This detection, however, is approximate because the counting of CFUs on Petri dish has a limit of detectability equal to $100 \mathrm{CFU}$.

Figure $9 \mathrm{~b}$ shows the number of surviving microorganisms $\mathrm{S}$ (t) during the plasma phase.

Also with regard to Argon, the decay of the surviving microorganisms during the phase of plasma, from 5 and 10 minutes, is modeled by means of an average mortality rate $\alpha_{\mathrm{av}}$, obtained as combination of the mortality rates $\alpha_{2}+\alpha_{3}$ and $\alpha_{2}+\alpha_{3^{*}}$, related to the action of UV radiation and charged particles, for faster and slower kinetic respectively.

After the time $\mathrm{t}$ for $5 \leq \mathrm{t}<10$, one can find:

$$
S(t)=S_{0} e^{-\alpha_{a v} t}
$$

with $\alpha_{\mathrm{av}}=1.4$.

\section{DISCUSSION: COMPARISON BETWEEN $\mathrm{H}_{2} \mathrm{O}_{2}$ AND ARGON}

From the results, differences emerge between plasma of Peroxide and plasma of Argon, both for the mechanisms of achievement of sterility and for time necessary to obtain the conditions required by WHO (reduction of bacterial load in a half cycle equal to $6 \log$ units).

The sterilizing action in case of peroxide is mainly related to the etching by the active species $\mathrm{OH}, \mathrm{H}$ and mostly $\mathrm{O}$, whereas in case of Argon an important role is played by UV radiation (with a higher photon concentration), and the etching is due to $\mathrm{Ar}+$ ion (the $\mathrm{OH}$ concentration remains the same for the two cases). The difference between the survival curves is shown in Figure 10.

The figure shows a more effective sterilizing action of Argon plasma. These results will be validated by an appropriate statistic, also by considering more resistant microorganisms such as spores.

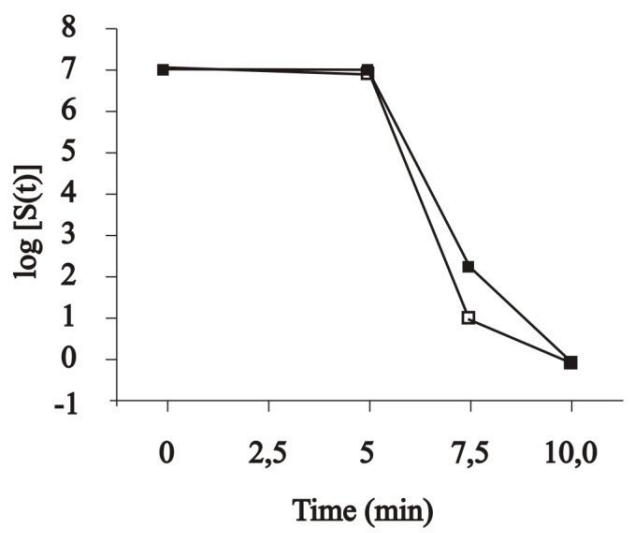

Figure 10. Survival curves of Escherichia coli subjected to the sterilization process with $\mathrm{H}_{2} \mathrm{O}_{2}$ (full symbols) and Argon (empty symbols) plasma
The action of the plasma consists in etching the membranes cellular, with external exposure of biological components such as proteins and DNA for subsequent inactivation by UV radiation. In case of the presence of argon and microorganisms are significant the appearance of nitrogen, a sign of etching of cellular components such as proteins or DNA, and that of carbon monoxide, a sign of etching of cell membranes. The disappearance of the $\mathrm{CO}$ emission line in the first 2.5 minutes of treatment could imply that the erosion of cell membranes is already exhausted in this time interval, in which nitrogen is still present but to a lesser extent. After this first-time interval, UV can directly affect the genetic material exposed to the outside thanks to the action carried out during the etching phase and exceed the intrinsic limit of the low penetration depth of UV radiation.

\section{CONCLUSIONS}

In this work, we described a plasma reactor, equipped with an extended set of plasma diagnostics, dedicated to the understanding the role of different agents acting in the sterilization process with gas plasma. We performed analysis in order to refer the killing of the bacteria only to the dissociation of the precursor gas and not to thermal effects (in fact we selected the power by maintaining the temperatures below the maximum value currently reached in the devices on the market) or to the effect of the not dissociated gas (in fact, in case of peroxide, we evaluated the effect of diffusion of the gas into the chamber). We present preliminary results on microbiological populations, obtained with hydrogen peroxide and Argon as precursor gases. Main mechanisms to achieve sterility conditions are identified and survival curves are considered in case of Escherichia Coli populations. Differences emerge between Peroxide and Argon plasma, for action mechanisms and sterilization time. The survival curves show a more effective sterilizing action of Argon plasma. A validation is necessary by an appropriate statistic and by considering more resistant microorganisms. The use of noncorrosive and non-irritating gas, such as argon, for sterilization purpose implies a simplification of the plant equipment. It would be possible to remove the components for the vaporization and concentration of peroxide, and to eliminate the management of the boxes containing discarded peroxide, managed to date as hazardous wastes and to reduce significantly the risk for health professionals. Currently, in the healthcare panorama there are many sterilization processes available to hospitals or research facilities. By classifying existing processes according to the temperature reached by the cycle, we have high temperature processes (Steam sterilization) and low temperature (Plasma sterilization, radiation, Ethylene Oxide and Peracetic Acid).

The use of low-temperature processes was made necessary by the spread of the intensive use of polymeric and thermolabile materials. Plasma sterilization perfectly meets this need, by not inducing changes in the properties of the materials subjected to treatment such as radiation, overcoming the problem of poor penetration of UV radiation and not involving any safety risk for operators as happens with ethylene oxide. The cycle used in autoclaves currently on the market involves the use of oxygen peroxide, an oxidizing and corrosive gas. The use of a non-corrosive and non-irritating gas would involve an important innovation. 
In this work a comparison is made between argon and hydrogen peroxide that currently is the only gas-plasma used in hospitals for sterilization purposes.

\section{REFERENCES}

[1] Bennett, J.E., Dolin, R., Blaser, M.J. (2014). Principles and Practice of Infectious Diseases. Philadelphia: Elsevier, 2014.

[2] Spaulding, E.H. (1972). Chemical disinfection and antisepsis in the hospital. Journal of Hospital Research, 9(1): 5-31.

[3] Klapes, N.A., Vesley, D. (1990). Vapor-phase hydrogen peroxide as a surface decontaminant and sterilant. Appl. Environ. Microbiol, 56(2): 503-4506.

[4] Radl, S., Ortner, S., Sungkornm, R., Khinast, J.G. (2009). The engineering of hydrogen peroxide decontamination systems. Journal of Pharmaceutical Innovation, 4: 51-62. https://doi.org/10.1007/s12247-009-9057-3

[5] Laroussi, M. (2005). Low temperature plasma-based sterilization: overview and state-of-the-art. Plasma Processes $\quad$ Polym., 2(5): 391-400. https://doi.org/10.1002/ppap.200400078

[6] Kyi, M.S., Holton, J., Ridgway, G.L. (1995). Assessment of the efficacy of a low temperature hydrogen peroxide gas plasma sterilization system. J. Hosp. Infect., 31(4): 275-284. https://doi.org/10.1016/0195-6701(95)90206-6

[7] Krebs, M.C., Bécasse, P., Verjat, D., Darbord, J.C. (1998). Gas-plasma sterilization: relative efficacy of the hydrogen peroxide phase compared with that of the plasma phase. Int. J. of Pharmaceutics, 160(1): 75-81. https://doi.org/10.1016/S0378-5173(97)00296-2

[8] Diab-Elschahawi, M., Blacky, A., Bachhofner, N., Koller, W. (2010). Challenging the Sterrad 100NX sterilizer with different carrier materials and wrappings under experimental 'clean' and 'dirty' conditions. Am J Infect Control., 38(10): 806-810. https://doi.org/10.1016/j.ajic.2010.05.023

[9] Venugopalan, M., Shih, A.L. (1981). Reactions of hydrogen peroxide vapor dissociated in a microwave plasma. Plasma Chemistry and Plasma Processing, 1: 191-200. https://doi.org/10.1007/BF00564580

[10] Vasko, C.A., Liu, D.X., Van Veldhuizen, E.M., Iza, F., Bruggeman, P.J. (2014). Hydrogen peroxide production in an atmospheric pressure RF glow discharge: Comparison of models and experiments. Plasma Chemistry and Plasma Processing, 34: 1081-1099. https://doi.org/10.1007/s11090-014-9559-8

[11] Shibata, T., Nishiyama, H. (2012). Decomposition of methylene blue in water using mist flow plasma reactor. Int. J. of Plasma Environmental Science \& Technology, 6(3): 253-259.

[12] Lee, H.W., Lee, H.W., Kang, S.K., Kim, H.Y., Won, I.H., Jeon, S.M., Lee, J.K. (2013). Synergistic sterilization effect of microwave-excited nonthermal Ar plasma, $\mathrm{H}_{2} \mathrm{O}_{2}, \mathrm{H}_{2} \mathrm{O}$ and $\mathrm{TiO}_{2}$, and a global modeling of the interactions. Plasma Sources Science and Technology., 22(5): 055008

[13] O’Connor, N., Cahill, O., Daniels, S., Galvin, S., Humphreys, H. (2014). Cold atmospheric pressure plasma and decontamination. Can it contribute to preventing hospital-acquired infections? Journal of Hospital Infection, 88(2): 59-65. https://doi.org/10.1016/j.jhin.2014.06.015

[14] Mai-Prochnowa, A., Murphya, A.B., McLean, K.M., Kong, M.G., Ostrikov, K.K. (2014). Atmospheric pressure plasmas: Infection control and bacterial responses. International Journal of Antimicrobial Agents., 43(6): 508-517. https://doi.org/10.1016/j.ijantimicag.2014.01.025

[15] Shintani, H., Sakudo, A., Burke, P., McDonnell, G. (2010). Gas plasma sterilization of microorganisms and mechanisms of action. Exp Ther Med., 1(5): 731-738. https://dx.doi.org/10.3892\%2Fetm.2010.136

[16] Barni, R., Decina, A., Zanini, S., D'Orazio, A., Riccardi, C. (2016). Experimental investigation of hydrogen peroxide RF plasmas. Plasma Sources Science and Technology, 25(2): 025003. https://doi.org/0.1088/09630252/25/2/025003

[17] Fiebrandt, M., Hillebrand, B., Lackmann, J.W., Raguse, M., Moeller, R., Awakowicz, P., Stapelmann, K. (2018). Inactivation of $\mathrm{B}$. subtilis spores by low pressure plasma - influence of optical filters and photon/particle fluxes on the inactivation efficiency. J. Phys. D: Appl. Phys., 51(4): 045401.

[18] Sanchez-Castro, J., Valle, G., Barillas, L., RawsonAcuña, F.E., Asenjo, J., Mora, J., Vargas, V.I. (2017). Effectiveness and efficiency optimization study of oxygen and argon DC low pressure plasma, sterilization. 2017 16th Latin American Workshop on Plasma Physics (LAWPP) pp.

73-78. https://doi.org/10.1109/LAWPP.2017.8692205

[19] Belgacem, Z.B., CarreÂ, G., Charpentier, E., Le-Bras, F., Maho, T., Robert, E., Pouvesle, J.M., Polidor, F., Gangloff, S.C., Boudifa, M., Gelle, M.P. (2017). Innovative non-thermal plasma disinfection process inside sealed bags: Assessment of bactericidal and sporicidal effectiveness in regard to current sterilization norms. PLoS ONE, 12(6): e0180183. https://doi.org/10.1371/journal.pone.0180183

[20] Riccardi, C., Barni, R., De Colle, F., Fontanesi, M. (2000). Modeling and diagnostic of an SF/sub 6/ RF plasma at low pressure. IEEE Transactions on Plasma Science, 28(1): https://doi.org/10.1109/27.842923

[21] Smith, J.M. (1987). Introduction to Chemical Engineering Thermodynamics. McGraw-Hill, 1987.

[22] Goedheer, W.J. (2000). Lecture notes on radio-frequency discharges, dc potentials, ion and electron energy distributions. Plasma Sources Science and Technology, 9(4): 507-516.

[23] Van Laar, J.J. (1910). Über Dampfspannungen von binären Gemischen (The vapor pressure of binary mixtures), Z. Physik. Chem., 72: 723-751.

[24] Barni, R., Zanini, S., Riccardi, C. (2008). Diagnostics of reactive RF plasmas. Vacuum, 82(2): 217-219. https://doi.org/10.1016/j.vacuum.2007.07.003

[25] Fantz, U. (2006). Basics of plasma spectroscopy. Plasma Sources Sci. Technol., 15(4): S137-147.

[26] Pearse, R.W.B., Gaydon, A.G. (1976). The Identification of Molecular Spectra. Wiley, 1976.

[27] Ralchenko, Y., Kramida, A.E., Reader, J. (2008). NIST Atomic Spectra Database. Gaithersburg MD: National Institute of Standards and Technology NIST Team, 2008. https://doi.org/10.18434/T4W30F

[28] Danil, D., Fridman, G., Friedman, G. (2009). Fridman, A., Physical and biological mechanisms of direct plasma 
interaction with living tissue. New Journal of Physics, 11: 115020.

[29] Vratnica, Z., Vujosevic, D., Cvelbar, U., Mozetic, M. (2008). Degradation of bacteria by weakly ionized highly dissociated radio-frequency oxygen plasma. IEEE Transactions on Plasma Science, 36(4): 1300-1301. https://doi.org/10.1109/TPS.2008.926841

[30] Philip, N., Saoudi, B., Crevier, M.C., Moisan, M., Barbeau, J., Pelletier, J. (2002). The respective roles of UV photons and oxygen atoms in plasma sterilization at reduced gas pressure: the case of $\mathrm{N}_{2}-\mathrm{O}_{2}$ mixtures. IEEE Transactions on Plasma Science, 30(4): 1429-1436. https://doi.org/10.1109/TPS.2002.804203

[31] Garcia De Castro, A., Bredholt, H., Strøm, A., Tunnacliffe, A. (2000). Anhydrobiotic engineering of gram negative bacteria. Applied and Environmental Microbiology, 66(9): 4142-4144. https://dx.doi.org/10.1128\%2Faem.66.9.41424144.2000

[32] Yu, H., Perni, S., Shi, J.J., Wang, D.Z., Kong, M.G., Shama, G. (2006). Effects of cell surface loading and phase of growth in cold atmospheric gas plasma inactivation of Escherichia coli K12. J. of Appl. Microbiology, 101(6): 1323-1330. https://doi.org/10.1111/j.1365-2672.2006.03033.x

[33] Muranyi, P., Wunderlich, J., Heise, M. (2008). Influence of relative gas humidity on the inactivation efficiency of a low temperature gas plasma. J. of Appl. Microbiology, 104(6): 1659-1666. https://doi.org/10.1111/j.13652672.2007.03691.x

[34] Roth, S., Feichtinger, J., Hertel, C. (2010). Characterization of Bacillus subtilis spore inactivation in low-pressure, low-temperature gas plasma sterilization processes. J. of Appl. Microbiology, 108(2): 521-531. https://doi.org/10.1111/j.1365-2672.2009.04453.x

[35] Roth, S., Feichtinger, J., Hertel, C. (2010). Response of Deinococcus radiodurans to low-pressure lowtemperature plasma sterilization processes. J. of Appl. Microbiology, 109(5): 1521-1530 https://doi.org/10.1111/j.1365-2672.2010.04771.x

[36] Muranyi, P., Wunderlich, J., Langowski, H.C. (2010). Modification of bacterial structures by a low-temperature gas plasma and influence on packaging material. J. of
Appl. Microbiology, 109(6) $1875-1885$ https://doi.org/10.1111/j.1365-2672.2010.04815.x

[37] Bourke, P., Ziuzina, D., Han, L., Cullen, P.J., Gilmore, B.F. (2017). Microbiological interactions with cold plasma. J. of Appl. Microbiology, 123(2): 308-324. https://doi.org/10.1111/jam.13429

[38] Puligundla, P., Mok, C. (2017). Potential applications of nonthermal plasmas against biofilm-associated microorganisms in vitro. J. of Appl. Microbiology, 122(5): 1134-1148. https://doi.org/10.1111/jam.13404

\section{NOMENCLATURE}

$\begin{array}{ll}\mathrm{CFU} & \text { Colony Forming Unit } \\ \mathrm{f} & \text { frequency of RF excitation } \\ \mathrm{P} & \text { power of RF excitation } \\ \mathrm{RF} & \text { Radio Frequency } \\ \mathrm{S} & \text { number of surviving microorganisms } \\ \mathrm{UV} & \text { Ultra Violet radiation } \\ \mathrm{VUV} & \text { Vacuum Ultra Violet radiation } \\ \mathrm{T}_{\mathrm{cmax}} & \text { maximum temperature currently reached in } \\ & \text { the commercial devices } \\ \mathrm{T}_{\mathrm{b}} & \text { maximum temperature at which bacteria } \\ & \text { can survive for a limited time }\end{array}$

\section{Greek symbols}

$\alpha \quad$ mortality rate

$\alpha_{1} \quad$ cellular mortality due to the action of the peroxide

$\alpha_{2} \quad$ cellular mortality related to the action of UV radiation

$\alpha_{3} \quad$ cellular mortality related to the action of charged particles

\section{Subscripts}

$\begin{array}{ll}\text { av } & \text { average } \\ \text { b } & \text { bacterial } \\ c & \text { commercial } \\ \text { exp } & \text { exposure } \\ \max & \text { Maximum value }\end{array}$

